\title{
ROTURA DE UN ANEURISMA ESPLÉNICO DURANTE EL EMBARAZO: A PROPÓSITO DE 2 CASOS CĹNICOS
}

\author{
Ernesto Perucca P. ${ }^{1}$, Jorge Cornejo J. ${ }^{1}$, Carlos Ruiz V. ${ }^{1}$, Jorge Castillo A. ${ }^{2}$, Boris León R. ${ }^{a}$, \\ Mariana Labbé T.a, Isabel Galleguillos F.a, Muschi Sziguetti Q. ${ }^{a}$ \\ ${ }^{1}$ Servicio de Obstetricia y Ginecología, ${ }^{2}$ Servicio de Anatomía Patológica Hospital Barros Luco-Trudeau.
}

a Internos de Medicina, Universidad de Chile, Facultad de Medicina, Sede Sur.

\section{RESUMEN}

Se presentan 2 casos de rotura de aneurisma de la arteria esplénica ocurridos a las 35 y 22 semanas de gestación. Una de las pacientes falleció por hemoperitoneo masivo.

PALABRAS CLAVES: Aneurisma de la arteria esplénica, hemoperitoneo, muerte materna

\section{SUMMARY}

We present two cases of splenic artery aneurysm rupture in pregnancies of 35 and 22 weeks of gestational age. One patient died because of massive hemoperitoneum.

\section{KEY WORDS: Splenic artery aneurysm, hemoperitoneum, maternal death}

\section{INTRODUCCIÓN}

La arteria esplénica es el tercer sitio más común de los aneurismas abdominales, la anteceden los aneurismas de la aorta y de las arterias iliacas (1). La verdadera prevalencia se desconoce; en autopsias se ha reportado rangos de 0,01 a $10,4 \%$, y se han encontrado en el $0,78 \%$ de los angiogramas $(1,2)$. Además se presenta en el $7,1 \%$ de los pacientes con hipertensión portal cirrótica (2). La ateroesclerosis es raramente la causa primaria, y por lo general es el resultado de la degeneración de la capa media de las arterias (2).

Esta entidad clínica fue descrita por primera vez por Beussier en 1776, y Corson en 1869 la describió durante el embarazo $(1,3)$. Si bien es cierto, la rotura de un aneurisma de la arteria esplénica ocurre en un 3 a $9 \%$ de los casos, la mitad de esta complicación acontece durante el embarazo (1, 2). La mortalidad materna alcanza alrededor de un $75 \%$ y la mortalidad fetal hasta un
$95 \%(1,2,3,4,5)$, complicándose más frecuentemente durante el tercer trimestre $(69 \%)(1,2,3$, 4).

Hasta 1999 en la literatura inglesa se habían publicado alrededor de 100 casos de aneurismas de la arteria esplénica, y sólo en 14 sobrevivió la madre y el feto (2).

Presentamos dos casos clínicos de rotura de aneurismas de la arteria esplénica, uno de ellos con resultado de muerte materna.

\section{CASOS CLÍNICOS}

\section{Caso 1}

Paciente de 35 años, derivada desde el Hospital del Pino, donde consultó por un cuadro de 4 horas de evolución, caracterizado por lipotimia post almuerzo, sudoración y disnea, junto con dinámica uterina. En ese hospital, por tratarse de paciente obesa de 96 kilos, IMC de 39 y con el antecedente de diabetes gestacional, se le practica hemo- 
glucotest que informa en $236 \mathrm{mg} / \mathrm{dl}$. Se encontraba lúcida, con pulso de 156 por minuto, presión arterial de 104/49 mmHg, y LCF de 152 por minutos, no pesquisándose dinámica uterina. Se deriva al Servicio de Obstetricia y Ginecología del Hospital Barros Luco-Trudeau para su estudio y manejo, con los diagnósticos de multípara de 4, embarazo de 35 semanas, obesidad, diabetes gestacional, lipotimia. Al ingreso se constató paciente con sensación vertiginosa, disnea y sudoración, además refiere dinámica uterina y disminución de los movimientos fetales. En el examen de ingreso estaba lúcida, orientada, piel y mucosas hidratadas y rosadas, pulso 80 por minuto, presión $80 / 40 \mathrm{mmHg}$, temperatura axilar de $36,7^{\circ} \mathrm{C}$. Examen físico segmentario normal. Cuello uterino sin modificaciones. Se ingresó a prepartos, con indicaciones de reposo absoluto, régimen de diabético, hidratación parenteral, y toma de glicemia. El estudio de la unidad fetoplacentaria, con registro basal no estresante es reactivo, pero se evidencia dinámica uterina indolora de 5 en 10 minutos. La ecografía obstétrica mostró feto único vivo, en cefálica, líquido amniótico normal, placenta normoinserta, anatomía fetal normal; estimación ecográfica de peso fetal de $2200 \mathrm{~g}$, en percentil 10-25, perfil biofísico de 8 puntos y doppler fetal normal.

La paciente permanece estable con presión arterial de 100/70 mmHg, hemoglucotest de 89 $\mathrm{mg} / \mathrm{dl}$. Es evaluada en numerosas oportunidades, hasta que a las 20:55 horas en que se encuentra disneica, lúcida, orientada, algo pálida con presiones de 100/70 mmHg, pulso de 88 por minuto, destaca la aparición de omalgia. Examen cardiopulmonar normal; abdomen blando, depresible, indoloro con Blumberg ausente; útero relajado, sin metrorragia. Se solicitó evaluación por medicina interna que sugiere ECG. Se estaba en estos trámites cuando a las 22:00 horas presentó brusco compromiso del estado general, sudoración, angustia, palidez acentuada y disnea en aumento, presión arterial no auscultable y ecografía obstétrica que mostró bradicardia fetal, por lo que se indica cesárea de urgencia. Al ingreso a pabellón a las 22:05 horas presenta paro cardiorrespiratorio, se realizan maniobras de reanimación e intubación. Se realiza laparotomía de urgencia, pesquisándose hemoperitoneo de $500 \mathrm{cc}$ en cavidad abdominal; se extrae recién nacido masculino con $2.460 \mathrm{~g}, 48$ $\mathrm{cm}$, circular al cuello y Apgar 0,3 y 4 , que requiere intubación y masaje cardíaco y traslado a ventilación mecánica en la UTI neonatal. Cirujano residente realiza masaje transdiafragmático recuperando la paciente ritmo sinusal de 140 por minuto, se explora la cavidad abdominal encontrándose a nivel de la transcavidad de los epiplones gran cantidad de coágulos de $2000 \mathrm{cc}$ aproximadamente y a nivel de hileo esplénico sangrado activo, por lo que se sospecha aneurisma esplénico roto y se realiza esplenectomía. Dada las malas condiciones de la paciente se deja con laparotomía contenida con compresas en lecho esplénico.

Persiste hipotensa pese a recuperación de ritmo sinusal y uso de coloides y cristaloides (6 litros), más 6 unidades de sangre total, junto a adrenalina, lidocaína y bicarbonato, por lo que se inicia administración de dopamina endovenosa logrando presiones en rango de 70/40, se pesquisa midriasis y ausencia de reflejo corneal. Se mantiene en respiración asistida, pulso filiforme de 140 por minuto, adecuada perfusión distal, pero con tendencia a la hipotensión. Fallece a las 1:40 horas del 21-02-01. Los diagnósticos terminales fueron shock hipovólemico, paro cardiorespiratorio prolongado, coma bulbar, aneurisma de la arteria esplénica rota. El estudio biópsico demostró bazo indemne y aneurisma roto de la arteria esplénica (Figuras 1 y 2).

El recién nacido de 35 semanas, pretérmino adecuado a la edad gestacional, evolucionó con asfixia neonatal severa prolongada, evolucionó desfavorablemente con edema cerebral, síndrome convulsivo y enfermedad de membrana hialina, coma profundo, arreflexia, con insuficiencia renal, disfunción miocárdica, trastornos de la coagulación, falleciendo a los 7 días de vida.

\section{Caso 2}

Paciente de 35 años que ingresó al Servicio de Obstetricia y Ginecología el 10 de septiembre

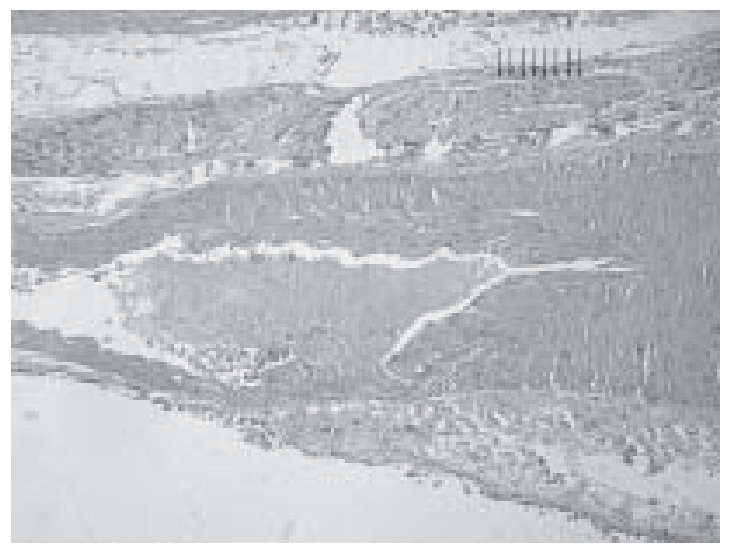

Figura 1. Caso 1. Dilatación y adelgazamiento aneurismático pre-rotura (ver $\downarrow$ ). 


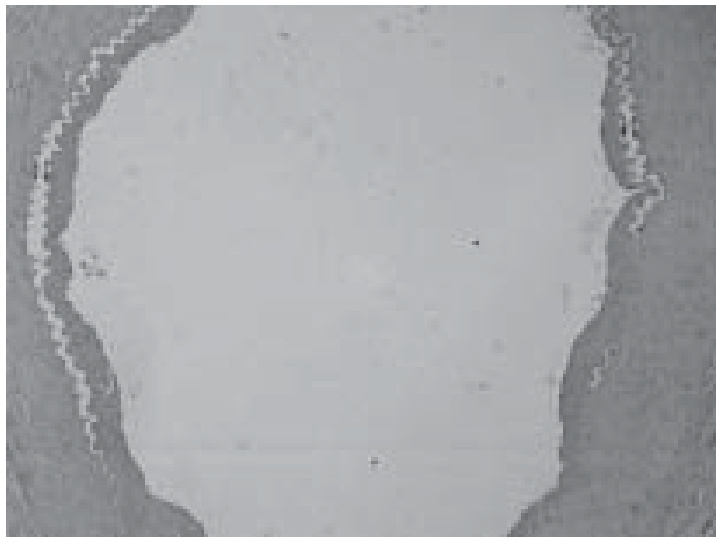

Figura 2. Caso 1. Degeneración quística de la túnica media del aneurisma de arteria esplénica (ver $\downarrow$ ).

de 2005 a las 22:05 horas enviada desde el Hospital de Buin con diagnóstico de multípara de 1, cesárea anterior, embarazo de 22 semanas por fecha de última menstruación, acorde con ecografía de las 9 semanas. Es derivada por cuadro de dolor abdominal de aproximadamente 12 horas de evolución, acompañado de fiebre y asociado a náuseas y vómitos. Al ingreso se describe paciente en condiciones generales satisfactorias, lúcida, rosada, pulso de 96 por minuto, $37,5^{\circ} \mathrm{C}$ axilar $\mathrm{y}$ $38^{\circ} \mathrm{C}$ rectal, $110 / 70$, altura uterina $20 \mathrm{~cm}$, latidos cardiofetales en 150 por minuto, sin metrorragia, con sonda nasogástrica in situ. El examen cardiopulmonar es semiológicamente normal y el abdomen se encuentra con discreto dolor difuso, Blumberg ausente y ruidos hidroaéreos presentes. La evolución de ingreso no impresiona como cuadro quirúrgico por lo que se deja en observación,

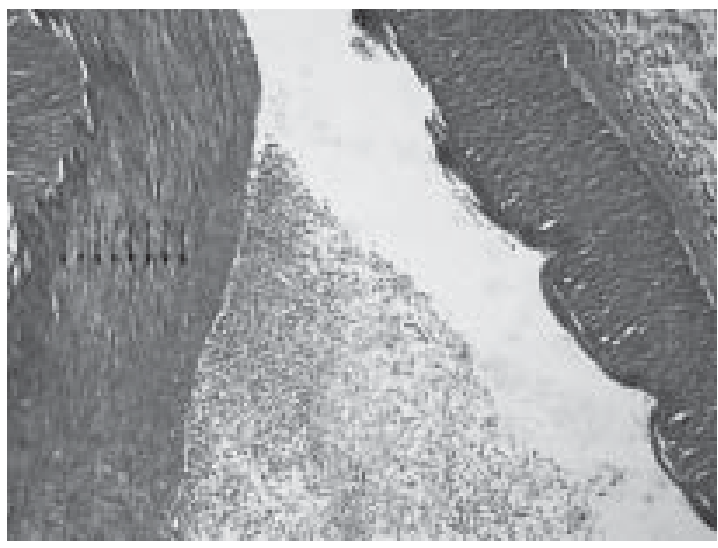

Figura 3. Caso 2. Engrosamiento irregular de la pared vascular (ver $\downarrow$ ). Displasia fibromuscular de la arteria esplénica.

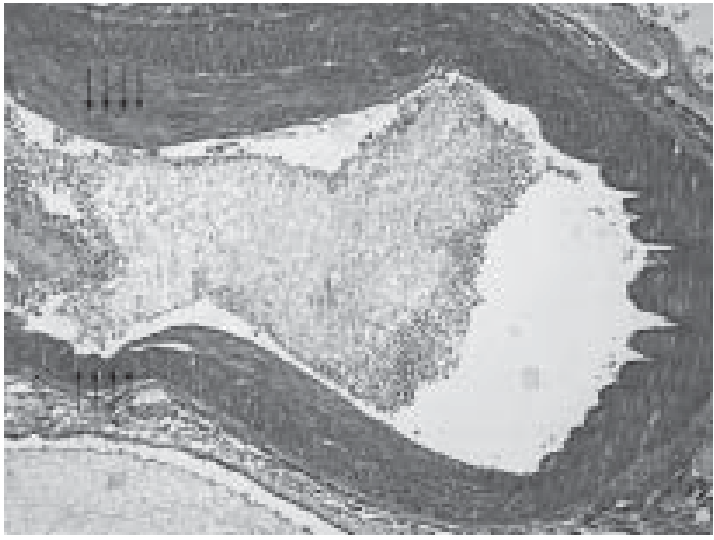

Figura 4. Caso 2. Engrosamiento irregular de la pared en relación a la íntima (displasia fibromuscular) (ver $\downarrow$ ). Zona de adelgazamiento de la pared (ver $\uparrow$ ).

con sonda nasogástrica, hidratación parenteral, solicitando evaluación por cirujanos. Se solicita recuento de leucocitos, PCR, pruebas hepáticas, lipasa y amilasa.

La ultrasonografía describe feto único vivo, frecuencia cardiaca de 162 por minutos, anatomía fetal normal, líquido amniótico normal, placenta anterior oclusiva y estimación de peso fetal de 570 g. Vista por cirujano tampoco le impresiona como cuadro quirúrgico. La paciente permanece estable con discreto dolor abdominal, pulso 84 por minutos, temperatura axilar de $37,2^{\circ} \mathrm{C}$ y rectal de $37,3^{\circ} \mathrm{C}, 110 / 70$. Abdomen con dolor discreto y difuso, y sin signos peritoneales.

Nueva evaluación al día siguiente a las 10:30 horas, ciclo vital sin variación, pero con evidente dolor a la palpación en fosa iliaca derecha y

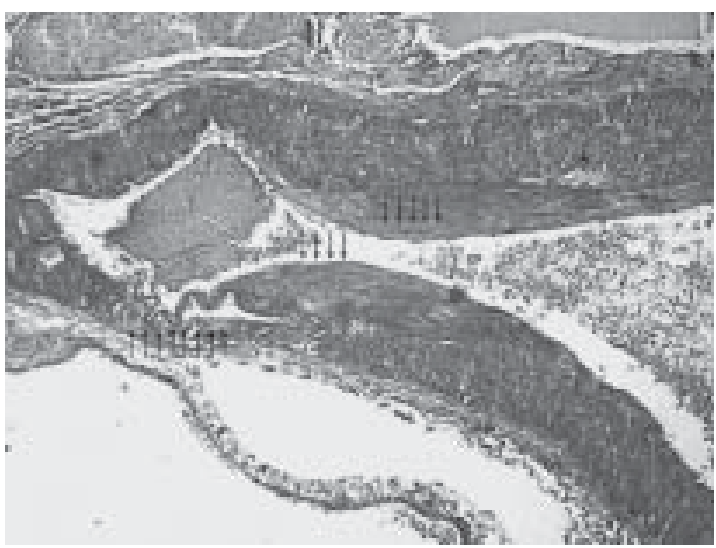

Figura 5. Caso 2. Displasia fibromuscular (ver $\downarrow$ ). Dilatación aneurismática en vías de rotura (ver $\uparrow$ ). 
Blumberg positivo, por lo que se decide realizar laparotomía exploradora, encontrándose hemoperitoneo de $1.500 \mathrm{cc}$, apéndice cecal sano, útero grávido normal y anexos normales, a la exploración se visualiza sangrado activo en hilio de la arteria esplénica, con bazo indemne, restos de órganos abdominales normales. Se práctica esplenectomía, trasladándose en el postoperatorio a la UTI, donde evolucionó favorablemente. En esta unidad se continuo con esquema antibiótico de metronidazol y ceftriazona a dosis terapéutica, posteriormente es trasladada a Maternidad donde continuó su recuperación y es dada de alta el 23/09/05 previa ecografía obstétrica que demostró evaluación fetal normal.

La biopsia de la pieza operatoria mostró bazo de $90 \mathrm{~g}$, cápsula lisa, sin desgarro, angiodisplasia arteria esplénica con microaneurisma secundario (Figuras 3, 4 y 5).

Posteriormente, la paciente es reenviada desde el Hospital de Buín al Servicio de Urgencia del Hospital Barros Luco Trudeau el 8 de octubre por cuadro franco de abdomen agudo, se realiza laparotomía exploradora en busca de una causa no obstétrica asociada al primer episodio, encontrándose alrededor de 2 litros de hemoperitoneo, la exploración de la cavidad abdominal era normal. Por este motivo se solicita la concurrencia de residente de obstetricia, quien encuentra una rotura uterina a nivel de segmento por placenta percreta. Se procede a la extracción de recién nacido femenino de $998 \mathrm{~g}$, Apgar 6 y 7 a los 5 minutos. Se continuó la cesárea con la histerectomía total con placenta in situ, debiéndose realizar anexectomía bilateral, dado que estaban infiltrados los anexos, además de un sangramiento en napa del lecho vesical que obligó a un packing de compresas en esa región y bolsa de Bogotá. Debió recibir 6 unidades de crioprecipitados, 5 unidades de plasma y 2 unidades de glóbulos rojos. Posteriormente es trasladada a la $\mathrm{UCl}$, donde evoluciona en buenas condiciones con apoyo de fluidos y antibióticos (ceftriazona y metronidazol), no presentando parámetros sépticos. Se realiza extracción de packing de compresas, y se procede a la laparorrafia el 10 de octubre, el 11 de octubre se realiza TAC que estaba dentro de límites normales, se traslada la paciente al servicio de Obstetricia y Ginecología donde continuó su evolución favorable, siendo dada de alta el día 21 de octubre. El recién nacido continua hospitalizado con evolución favorable, presentó membrana hialina y la ecografía craneana mostró hemorragia intracraneana grado 1 a 2. La biopsia del útero mostró placenta acreta.

\section{CONCLUSIONES}

Aunque la rotura de un aneurisma de la arteria esplénica es una entidad poco frecuente, es potencialmente catastrófica, con una alta mortalidad materna y perinatal. El tratamiento debe estar limitado a aneurismas cuyo diámetro es mayor o igual a $2 \mathrm{~cm}$, pero en la mujer en edad reproductiva debería ser tratado en forma electiva, debido al incremento del riesgo de rotura durante la gestación de hasta un $50 \%$, en contraste con la baja mortalidad de 0,5 a $1,3 \%$ de la cirugía electiva.

El diagnóstico por sí es difícil y debe ser considerado en cualquier embarazada que presenta dolor en el cuadrante superior izquierdo del abdomen, asociado o no a shock hipovolémico. El diagnóstico diferencial debe hacerse con desprendimiento prematuro de placenta, rotura uterina, úlcera gástrica perforada o rotura de otro aneurisma arterial.

Se ha descrito un fenómeno de doble ruptura que es reportado en $20 \%$ de los casos. La paciente presenta un repentino dolor abdominal e hipotensión, el cual se recupera rápidamente con reemplazo simple de fluidos, posteriormente hay un período de latencia seguido de un brusco colapso cardiovascular que sucede dentro de las primeras 24 horas.

En nuestra muerte materna no fue sospechada la rotura del aneurisma esplénico y se presentó el fenómeno de doble ruptura; dada su rareza constituyó un hallazgo en la laparotomía, aunque la presentación clínica fue muy similar a los cuadros clínicos descritos en la literatura.

\section{BIBLIOGRAFÍA}

1. Holdsworth R, Gunn A. Ruptured splenic artery aneurysm in pregnancy. A review. $\mathrm{Br} \mathrm{J}$ Obstet Gynaecol 1992; 99: 595-7.

2. Selo-Ojeme D, Welch C. Spontaneous rupture of splenic artery aneurysm in pregnancy. Europ J Obstet Gynecol Reprod Biol 2003; 109: 124-7.

3. De Perrot M, Buhler L, Deléaval J, Boris B, Mentha $G$, Morel P. Management of true aneurysm of the splenic artery. Am J Surg 1998; 175: 466-8.

4. Herbeck M, Horbach T, Putzenlechner C, Klein P, Lang W. Ruptured splenic artery aneurysm during pregnancy: A rare case with both maternal and fetal survival. Am J Obstet Gynecol 1999; 181: 763-4.

5. Fender G, Haslett E, Chir B, Leary T, Bland E, Hackett G. Management of splenic artery aneurysm rupture during trial of scar with epidural analgesia. Am J Obstet Gynecol 1999; 180: 1038-9. 